\title{
GENERALIZED TWO DIMENSIONAL CANONICAL TRANSFORM
}

\author{
S.B.Chavhan \\ Yeshawant Mahavidhalaya Nanded (India)
}

\begin{abstract}
The two-dimensional canonical transform can be used in optical system analysis, image processing and pattern recognition. In this paper two-dimensional transform anonical is extended to the distribution of compact support. Analyticity theorem, inversion theorem, is proved for this transform. Lastly properties of kernel are discussed.
\end{abstract}

Keyword:- Canonical transform, two-dimensional canonical transform Generalized function, signal processing.

\section{Introduction:}

Now a days fractional integral transform play an important role in signal processing, image reconstruction, pattern recognition, acoustic signal processing,[1], [2].The fractional Fourier transform[3], [4], which is the generalization of

the one-dimensional Fourier transforms is defined as

$$
F^{\alpha}(s)=\sqrt{\frac{1-\cot \alpha}{2 \pi}} e^{\frac{i}{2}\left(\cot \alpha \cdot s^{2}\right)} \int_{-\infty}^{\infty} e^{-i \cot \alpha t^{2}} e^{\frac{i}{2} \cot \alpha t^{2}} f(t) d t \ldots \ldots \ldots
$$

It has the following additivity property

$$
o_{F}^{\beta} . o_{F}^{\alpha}=o_{F}^{\alpha+\beta}
$$

In fact, the fractional Fourier transform is the special case of the canonical transform [5],[6]. The canonical transform is defined as

$$
\therefore\{2 D C T f(t, x)\}(s, w)=\frac{1}{\sqrt{2 \pi i b}} e^{\frac{i}{2}\left(\frac{d}{b} s^{2}\right.} \int_{-\infty}^{\infty} e^{-i\left(\frac{s}{b} t\right)} e^{\frac{i}{2}\left(\frac{a}{b}\right) t^{2}} f(t) d t \mathrm{~b} \neq 0
$$

The one-dimensional canonical transform can be extended in to two-dimensional canonical transform as follows. $\therefore\{2 D C T f(t, x)\}(s, w)$

$$
=\frac{1}{\sqrt{2 \pi i b}} \frac{1}{\sqrt{2 \pi i b}} e^{\frac{i}{2}\left(\frac{d}{b}\right) s^{2}} e^{\frac{i}{2}\left(\frac{d}{b}\right) w^{2}} \int_{-\infty}^{\infty} e^{-i\left(\frac{s}{b} t\right)} e^{-i\left(\frac{w}{b} x\right)} e^{\frac{i}{2}\left(\frac{a}{b}\right)^{2}} e^{\frac{i}{2}\left(\frac{a}{b}\right)^{2}} f(t, x) d x d t \quad \mathrm{~b} \neq 0
$$

Notation and terminology as per Zemanian [7].

This paper is organized as follows: Section 2 the definition two- dimensional canonical transform, and testing function space. Section 3 inversion and Analyticity theorem, are proved. Section 4 properties of kernel are discussed.

\section{Definition two dimensional (2D) canonical transform:}

Where we have, given the definition of two dimensional (2D) generalized canonical transform.

\subsection{Two-dimensional Generalized canonical transform $E^{\prime}(R \times R)$ :}

It can be easily proved that the functions $K_{f_{1}}(t, s)$ and $K_{f_{2}}(x, w)$ which are the functions of $t$ and $x$ are members of $E(R \times R)$.

where, 


$$
K_{f_{1}}(t, s)=\frac{1}{\sqrt{2 \pi i b}} e^{\frac{i}{2}\left(\frac{d}{b}\right) s^{2}} e^{\frac{i}{2}\left(\frac{a}{b}\right) t^{2}} \cdot e^{-i\left(\frac{s}{b} t\right)} \quad \text { and } \quad K_{f_{2}}(x, w)=\frac{1}{\sqrt{2 \pi i b}} e^{\frac{i}{2}\left(\frac{d}{b}\right) w^{2}} e^{\frac{i}{2}\left(\frac{a}{b}\right) x^{2}} \cdot e^{-i\left(\frac{w}{b} x\right)}
$$

$$
\text { That is } \gamma_{E, k}\left\{K_{f_{1}}(t, s) K_{f_{2}}(x, w)\right\}=\begin{aligned}
& \sup \\
& -\infty<t<\infty \\
& -\infty<x<\infty
\end{aligned}\left|D_{t}^{k} D_{x}^{l} K_{f_{1}}(t, s) K_{f_{2}}(x, w)\right|<\infty
$$

let $E^{\prime}(R \times R)$ denotes the dual of $E(R \times R)$. Therefore the generalized canonical transform of $f(t, x) \in E^{\prime}(R \times R)$ can be defined as

$$
\{2 D C T f(t, x)\}(s, w)=\left\langle f(t, x), K_{f_{1}}(t, s) K_{f_{2}}(x, w)\right\rangle
$$

$\therefore\{2 D C T f(t, x)\}(s, w)$

$$
=\frac{1}{\sqrt{2 \pi i b}} \frac{1}{\sqrt{2 \pi i b}} e^{\frac{i}{2}\left(\frac{d}{b}\right) s^{2}} e^{\frac{i}{2}\left(\frac{d}{b}\right) w^{2}} \int_{-\infty}^{\infty} e^{-i\left(\frac{s}{b} t\right)} \cdot e^{-i\left(\frac{w}{b} x\right)} \cdot e^{\frac{i}{2}\left(\frac{a}{b}\right) x^{2}} e^{\frac{i}{2}\left(\frac{a}{b}\right) t^{2}} f(t, x) d x d t
$$

Where $K_{f_{1}}(t, s)=\frac{1}{\sqrt{2 \pi i b}} e^{\frac{i}{2}\left(\frac{d}{b}\right) s^{2}} e^{-i\left(\frac{s}{b}\right) t} e^{\frac{i}{2}\left(\frac{a}{b}\right) t^{2}} \quad$ when $b \neq 0$

$$
\begin{array}{cc}
=\sqrt{d} e^{\frac{i}{2}\left(c d s^{2}\right)} \delta(t-d s) & \text { when } \mathrm{b}=0 \\
K_{f_{2}}(x, w)=\frac{1}{\sqrt{2 \pi i b}} e^{\frac{i}{2}\left(\frac{d}{b}\right) w^{2}} e^{-i\left(\frac{w}{b}\right) x} e^{\frac{i}{2}\left(\frac{a}{b}\right) x^{2}} & \text { when } b \neq 0 \\
=\sqrt{d} e^{\frac{i}{2}\left(c d w^{2}\right)} \delta(x-d . w) & \text { when } \mathrm{b}=0
\end{array}
$$

2.2 Definition of testing function space:

An infinitely differentiable complex valued function $\phi$ on $R^{n}$ belongs to $E\left(R^{n}\right)$, if for each compact set.

$$
\begin{aligned}
& I \subset s_{a}, J \subset s_{b} \text { where } \quad s_{a}=\left\{t: t \in R^{n}, \quad|t| \leq a, a>0\right\}, s_{b}=\left\{x: x \in R^{n}, \quad|x| \leq b, b>0\right\} \\
& \text { and for } I \in R^{n}, J \in R^{n}, \\
& \gamma_{E, k} \phi(t, x)=\sup _{\substack{-\infty<<<\infty \\
-\infty<<<\infty<}}\left|D_{t}^{k} D_{x}^{l} \phi(t, x)\right|<\infty \quad \mathrm{k}=0,1,2,3 . \text { and } l=0,1,2,3 \ldots
\end{aligned}
$$

Thus $E\left(R^{n}\right)$ will denotes the space of all $\phi(t, x) \in E\left(R^{n}\right)$ with support contained in $s_{a}$ and $s_{b}$. Note that space $\mathrm{E}$ is complete and a Frechet space, let $E^{\prime}$ denotes the dual space of $E$.

\section{Inversion and Analyticity of Two Dimensional canonical transform:}

\subsection{Inverse of Two Dimensional canonical transform:}

If $\{2 D C T f(t, x)\}(s, w)$ is canonical transform of $f(t, x)$ then inverse of transform is given by

$$
f(t, x)=\sqrt{\frac{2 \pi i}{b}} \sqrt{\frac{2 \pi i}{b}} e^{\frac{-i}{2}\left(\frac{a}{b}\right) t^{2}} e^{\frac{-i}{2}\left(\frac{a}{b}\right) x^{2}} \int_{-\infty}^{\infty} \int_{-\infty}^{\infty} e^{-i\left(\frac{s}{b} t\right)} e^{-i\left(\frac{w}{b} t\right)} e^{\frac{-i}{2}\left(\frac{d}{b}\right) s^{2}} e^{-\frac{i}{2}\left(\frac{d}{b}\right) w^{2}}\{2 D C T f(t, x)\}(s, w) d s d w
$$

\subsection{Analyticity theorem:}

Let $f \in E^{1}\left(R^{n}\right)$ and let its two canonical transform be defined by, 
$\{2 D C T f(t, x)\}(s, w)=\sqrt{\frac{1}{2 \pi i b}} e^{\frac{i}{2}\left(\frac{d}{b}\right) s^{2}} \sqrt{\frac{1}{2 \pi i b}} e^{\frac{i}{2}\left(\frac{d}{b}\right) w^{2}} \int_{-\infty}^{\infty} e^{\frac{i}{2}\left(\frac{a}{b}\right)^{2}} e^{\frac{i}{2}\left(\frac{a}{b}\right) x^{2}} e^{-i\left(\frac{s}{b}\right) t} e^{-i\left(\frac{w}{b}\right) x} f(t, x) d x d t$

then $\quad\{2 D C T f(t, x)\}(s, w) \quad$ is analytic $\quad$ on $\quad C^{n}$,if the $\quad$ a, $\quad$ b, sup $p f \subset s_{a}$ and $\quad s_{b}$ where $s_{a}=\left\{t: t \in R^{n}, \quad|t| \leq a, a>0\right\}, s_{b}=\left\{x: x \in R^{n}, \quad|x| \leq b, b>0\right\} \quad$ moreover $\quad\left\{\begin{array}{llll}2 D & C & T(\not), & t\end{array}\right) \quad$ is differentiable and $\quad D_{s}^{k} D_{w}^{l}\{2 D C T f(t, x)\}(s, w)=\left\langle f(t, x), D_{s}^{k} D_{w}^{l} K_{f_{1}}(t, s) K_{f_{2}}(x, w)\right\rangle$

Proof: Let, $s:\left\{s_{1}, s_{2}, \ldots \ldots \ldots s_{j} \ldots \ldots s_{n}\right\} \in C^{n}$ and $w:\left\{w_{1}, w_{2}, \ldots \ldots \ldots w_{j} \ldots \ldots w_{n}\right\} \in C^{n}$

We first prove that, $\frac{\partial}{\partial s_{j}} \frac{\partial}{\partial w_{j}}\{2 D C T f(t, x)\}(s, w)$ exists,

$$
\frac{\partial^{n}}{\partial s_{j}^{n}} \frac{\partial^{n}}{\partial w_{j}^{n}}\{2 D C T f(t, x)\}(s, w)=<f(t, x), \frac{\partial^{n}}{\partial s_{j}^{n}} \frac{\partial^{n}}{\partial w_{j}^{n}} K_{f_{1}}(t, s) K_{f_{2}}(x, w)>
$$

we prove the result $n=1$, the general result following by induction.

For fixed $s_{j} \neq 0$ choose two concentric circles $C$ and $C^{l}$ with centre $s_{j}$ and radii $r$ and $r_{l}$ respectively such that $0<r<r_{l}<\left|s_{j}\right|$.

Let $\Delta s_{j}$ be a complex increment satisfying $0<\left|\Delta s_{j}\right|<r$. Also for fixed $w_{j} \neq 0$.Again choose two concentric circles $C$ and $C_{1}$ with centre $w_{j}$ and radii $r^{\prime}$ and $r_{1}^{\prime}$ respectively such that $0<r^{\prime}<r_{1}^{\prime}<\left|w_{j}\right|$.

Let $\Delta w_{j}$ be a complex increment satisfying $0<\left|\Delta w_{j}\right|<r^{\prime}$

Consider,

$$
\begin{aligned}
\frac{(2 D C T)\left(s_{j}+\Delta s_{j}, w_{j}\right)-(2 D C T)\left(s_{j}, w_{j}\right)}{\Delta s_{j}} & \frac{(2 D C T)\left(s_{j}, w_{j}+\Delta w_{j}\right)-(2 D C T)\left(s_{j}, w_{j}\right)}{\Delta w_{j}} \\
& -<f(t, x), \frac{\partial}{\partial s_{j}} \frac{\partial}{\partial w_{j}} K_{f_{1}}(t, s) K_{f_{2}}(x, w)>
\end{aligned}
$$

$=<f(t, x), \Psi \Delta s_{j}(t) \Psi \Delta w_{j}(x)>$

where $\Psi \Delta s_{j}(t) \Delta w_{j}(x)=\frac{1}{\Delta s_{j}}\left[K_{f_{1}}\left(t, s_{1}, s_{2} \cdots \cdots s_{j}+\Delta s_{j} \cdots s_{n}\right)-K_{f_{1}}(t, s)\right]$

$\frac{1}{\Delta w_{j}}\left[K_{f_{2}}\left(x, w_{1}, w_{2} \cdots \cdots w_{j}+\Delta w_{j} \cdots w_{n}\right)-K_{f_{2}}(x, w)\right]-\frac{\partial^{n}}{\partial s_{j}^{n}} \frac{\partial^{n}}{\partial w_{j}^{n}} K_{f_{1}}(t, s) K_{f_{2}}(x, w)>$

For any fixed $(t, x) \in R^{n}$ and any fixed integer.

$k=\left(k_{1}, k_{2} \cdots \cdots k_{n}\right) \in N_{0}^{n} \quad$ and $\quad l=\left(l_{1}, l_{2} \cdots \cdots l_{n}\right) \in N_{0}^{n}$

$D_{t}^{k} D_{x}^{l} K_{f_{1}}(t, s) K_{f_{2}}(x, w)$ is analytic inside and on $C^{\prime}$ and $C_{1}^{\prime}$.

We have, by Cauchy integral formula. 


$$
\begin{aligned}
& D_{t}^{k} D_{x}^{l} \Psi \Delta s_{j} \Delta w_{j}(t, x)=\frac{1}{4 \pi^{2} i^{2}} D_{t}^{k} D_{x}^{l} K_{f_{1}}(t, s) K_{f_{2}}(x, w) \iint_{c_{1}}\left(\frac{1}{\Delta s_{j}}\left(\frac{1}{z-s_{j}-\Delta s_{j}}-\frac{1}{z-s_{j}}\right)-\frac{1}{\left(z-s_{j}\right)^{2}}\right) \\
& \quad\left(\frac{1}{\Delta w_{j}}\left(\frac{1}{y-w_{j}-\Delta w_{j}}-\frac{1}{y-w_{j}}\right)-\frac{1}{\left(y-w_{j}\right)^{2}}\right) d z d y \\
& =\frac{\Delta s_{j} \Delta w_{j}}{-4 \pi^{2}} \iint_{\bar{c}_{1}} \frac{D_{t}^{k} D_{x}^{l} K_{f_{1}}(t, \bar{s}) K_{f_{2}}(x, \bar{w})}{\left(z-s_{j}-\Delta s_{j}\right)\left(z-s_{j}\right)^{2}\left(y-w_{j}-\Delta w_{j}\right)\left(y-w_{j}\right)^{2}} d z d y
\end{aligned}
$$

where,

But for all $z \in C^{\prime}$ and $y \in C_{1}^{\prime}$ and $(t, x)$ restricted to a compact subset of $R^{n}$,

$$
\begin{gathered}
D_{t}^{k} D_{x}^{l} K_{f_{1}}(t, s) K_{f_{2}}(x, w) \text { is } \quad \text { bounded } \\
\left|D_{t}^{k} D_{x}^{l} \Psi \Delta s_{j} \Delta w_{j}(t, x)\right| \leq \frac{\left|\Delta s_{j}\right|\left|\Delta w_{j}\right|}{4 \pi^{2}} \iint_{c} \frac{Q}{c_{c_{1}}} \frac{Q}{\left(r_{1}-r\right)\left(r_{1}\right)\left(r_{1}^{\prime}-r\right)\left(r_{1}^{\prime}\right)}|d z||d y| \\
\leq \frac{\left|\Delta s_{j}\right|\left|\Delta w_{j}\right|}{4 \pi^{2}} \frac{Q}{\left(r_{1}-r\right)\left(r_{1}\right)\left(r_{1}^{\prime}-r\right)\left(r_{1}^{\prime}\right)}
\end{gathered}
$$

Thus as $\left|\Delta s_{j}\right| \rightarrow 0$, and $\left|\Delta w_{j}\right| \rightarrow 0, \quad D_{t}^{k} D_{x}^{l} \Psi \Delta s_{j} \Delta w_{j}(t, x)$ tends to zero. Uniformly on the compact subset of $R^{n}$.Therefore it follows that $\Psi \Delta s_{j} \Delta w_{j}(t, x)$ converges in $E\left(R^{n}\right)$ to zero since $f \in E^{1}$, we concluded (5) tends to zero. Therefore $\{2 D C T f(t, x)\}(s, w)$ is differentiable with respective $s_{j}$ and $w_{j}$. But this is true for all $\mathrm{i}$, $j=1,2, \ldots \ldots . .$. .Hence

$\{2 \operatorname{DCTf}(t, x)\}(s, w)$ is analytic on $C^{n}$ and,

$$
D_{s}^{k} D_{w}^{l}\{2 D C T f(t, x)\}(s, w)=<f(t, x), D_{s}^{k} D_{w}^{l} K_{f_{1}}(t, s) K_{f_{2}}(x, w)>
$$

\section{Properties of kernel:}

$$
\text { If }\{2 D C T f(t, x)\}(s, w)=\frac{1}{\sqrt{2 \pi i b}} \frac{1}{\sqrt{2 \pi i b}} e^{\frac{i}{(2}\left(\frac{d}{b}\right) s^{2}} e^{\frac{i}{2}\left(\frac{d}{b}\right) w^{2}} \int_{-\infty}^{\infty} \int_{-\infty}^{\infty} e^{-i\left(\frac{s}{b} t\right)} e^{-i\left(\frac{w}{b}\right) x} e^{\frac{i}{2}\left(\frac{a}{b}\right)^{2}} e^{\frac{i}{2}\left(\frac{a}{b}\right) x^{2}} f(t, x) d x d t
$$

is definition two dimensional canonical transform of $f(t, x)$

Where,

$$
\begin{array}{rlrl}
k_{f_{1}}(t, s), k_{f_{2}}(x, w) & =\frac{1}{\sqrt{2 \pi i b}} \frac{1}{\sqrt{2 \pi i b}} e^{\frac{i}{2}\left(\frac{d}{b}\right)^{s^{2}}} e^{\frac{i}{2}\left(\frac{d}{b}\right)^{w^{2}}} e^{-i\left(\frac{s}{b}\right) e^{-i\left(\frac{w}{b} x\right)} e^{\frac{i}{2}\left(\frac{a}{b}\right) t^{2}} e^{2} \frac{i}{2\left(\frac{a}{b}\right) x^{2}}} & \text { when } b \neq 0 \\
=\sqrt{d} e^{\frac{i}{2}\left(c d s^{2}\right)} \delta(t-d s) \sqrt{d} e^{\frac{i}{2}\left(c d w^{2}\right)} \delta(x-d w) & \text { when } \mathrm{b}=0
\end{array}
$$

kernel of 2D canonical transform satisfied following property
4.1) $k_{f_{1}}(t, s) k_{f_{2}}(x, w)=k_{f_{1}}(t,-s) k_{f_{2}}(x, w)$
4.2) $k_{f_{1}}(-t, s) k_{f_{2}}(-x, w)=k_{f_{1}}(t,-s) k_{f_{2}}(x,-w)$
4.3) $k_{f_{1}}(t, s) k_{f_{2}}(x, w)=k_{f_{1}}(s, t) k_{f_{2}}(w, x)$ if a $=\mathrm{b}$
4.4) $k_{f_{1}}(t, s) k_{f_{2}}(x, w)=k_{f_{1}}(s, t) k_{f_{2}}(w, x)$ if a $\neq b$

Five properties of kernel, stated above are simple to prove, hence the proof omitted. 


\section{Conclusion:}

The two-dimensional canonical transform is generalized in the distributional sense. Its inversion and Analyticity theorem is proved. Some properties of kernel are discussed. It can be used optical system analysis.

\section{References:}

[1] Tatiana Alieva and Bastianas Martin J., “On Fractional Fourier transform moments,” IEEE signal processing letters, Vol. 7, No.11, Nov. 2000.

[2] Tatiana Alieva and Bastiaans Martian J., "Wigner Distribution and Fractional Fourier Transform for 2-Dimentional Symmetric Beams", JOSA A, Vol.17, No.12, P.2319 - 2323, Dec.2000.

[3] L. B. Almeida. "The fractional Fourier transform and time frequency," representation IEEE trans. Signal Processing, Vol. 42, No. 11, Nov. 1994

[4] V. Namias., "The fractional order Fourier transform and its application to quantum mechanics," J. Inst. Math. Appticat., Vol. 25, pp. 241-265 (1980).

[5] M. Moshinky and C.Quesne.,Linear canonical transform and their unitary representation, J. math, Phy., Vol. 12, No. 8, P. 1772-1783, 1971.

[6] S.Abe and J.T.Sheridan., "Optical operations on wave function as the Abelian subgroups of the special affine Fourier transformation,”Opt.lett.,vol.19,no.22,pp.1801-1803,1994.

[7] Zemanian A.H., "Generalized integral transform," Inter Science Publisher's New York, 1968. 